\title{
Propuesta de indicadores de la Responsabilidad Social Universitaria conforme a la guía G4 del GRI: el caso de la Universidad de Córdoba
}

\author{
Clara Guijarro Jiménez \\ Antonio Gomera Martínez \\ Miguel Antúnez López
}

\section{RESUMEN}

El uso de indicadores resulta una herramienta útil de evaluación en el desarrollo de estrategias de Responsabilidad Social Universitaria (RSU). Además, el cumplimiento con estándares internacionales para la elaboración de memorias de responsabilidad social ayuda a crear una visión integrada de la contribución de las organizaciones a la sostenibilidad. Ambas prácticas son cada vez más comunes en el ámbito universitario nacional. El objetivo de este artículo es elaborar una propuesta de indicadores de la RSU acorde con la versión más reciente de la "Guía para la elaboración de memorias de sostenibilidad" del Global Reporting Initiative. Para ello se han analizado comparativamente las dos últimas versiones de dicha Guía y una muestra de memorias de RSU de universidades españolas, además de las distintas propuestas nacionales que tratan de integrar las dimensiones educacionales en este tipo de informes.

PALABRAS CLAVE: Indicador, Global Reporting Initiative, Responsabilidad Social, Universidad, sostenibilidad.

CLAVES ECONLIT: D83, I23, M14.

Cómo citar este artículo / How to cite this article: GUIJARRO, C., GOMERA, A. \& ANTÚNEZ, M. (2016): "Propuesta de indicadores de la Responsabilidad Social Universitaria conforme a la Guía G4 del GRI: el caso de la Universidad de Córdoba", CIRIEC-España, Revista de Economía Pública, Social y Cooperativa, 87, 103-137.

Correspondencia: Clara Guijarro Jiménez, Servicio de Protección Ambiental de la Universidad de Córdoba, e-mail: cguijarro@uco.es; Antonio Gomera Martínez, Servicio de Protección Ambiental de la Universidad de Córdoba, e-mail: agomera@uco.es, y Miguel Antúnez López, Aula de Sostenibilidad de la Universidad de Córdoba, e-mail: aulasostenibilidad@uco.es. 


\section{EXPANDED ABSTRACT}

\section{Proposal for University Social Responsibility indicators according to GRI G4 guidelines: the case of the University of Cordoba (Spain)}

\section{Aims and objectives}

Taking into account the key role of socially responsible management transparency and accountability in public universities in Spain, this article shows a framework proposal to write up social responsibility reports based on international standardized tools. In this way, this paper facilitates the comparability of the socially responsible initiatives and the analysis of their evolution over time.

The general purpose of this paper is to produce a list of performance indicators adapted to Spanish universities reality with an approach based on the most recent version of Global Reporting Initiative's Sustainability Reporting Guidelines.

In order to do so, the following specifics objectives have been identified:

- To identify social responsibility indicators in university reports according to the GRI G4 Sustainability Guidelines (the Guidelines).

- To analyze how this information adapts to the Guidelines performance indicators.

- To complete the list of indicators making a proposal that enable drawing up the future university social responsibility reports according to the GRI G4 Sustainability Guidelines.

\section{Methods}

In the context of public universities in Spain and university social responsibility, we have carried out a case study whose context is the University of Cordoba. This study has analyzed university social responsibility reports of the University of Cordoba for adapting them to the GRI G4 Sustainability Guidelines in the future.

Currently, Spanish universities choose models proposed by the GRI or by Social Council of Public Universities in Andalusia Forum; or may choose or a combination of both models, as the University of Cordoba in the last three reports.

After the comparative study of the last two versions of the GRI Guidelines (2011 and 2013), the next step focused on the University of Cordoba has been to analyze the information contained in 2011/2012 university social responsibility report. In that moment, we identified several performance indicators which complied with the GRI G4 Sustainability Guidelines' proposal on a greater or lesser degree. 
With the aim of identifying the most frequent indicators in Spanish public universities, we have used a sample of social responsibility reports from five Spanish universities and different national proposals which try to integrate the educational dimension in such reports. These universities already used the GRI 3.1 Sustainability Guidelines (2011) to draw up their reports, but not the GRI G4 (2013).

Once GRI 3.1 performance indicators of the University of Cordoba and the rest of universities of the sample were compiled, the next task was to make a selection from these indicators in order to make our proposal according to the following criteria:

- Indicators used by the University of Cordoba.

- Indicators adapted to the GRI G4 Sustainability Guidelines.

- The most commonly used indicators by the rest of universities in the sample.

Right after that, the content of each indicator was reviewed and adapted to the GRI G4 Sustainability Guidelines. For each proposal, we have identified its function and its justification.

Finally, we have verified our proposals through expert opinion.

\section{Results}

As an outcome of the investigation we have obtained a proposal of indicators which allows the University of Cordoba and the rest of public universities in Spain to draw up social responsibility reports according to the GRI G4 Sustainability Guidelines. Likewise, if we consider the international profile of the GRI G4 Sustainability Guidelines, on which this article is based, our proposal could be applied to international higher education institution.

This proposal for indicators has been divided in to three sections depending on whether it is economic, environmental or social indicator. The indicators in each section in turn are grouped together by 'aspects' or 'material topics' in the same order than they appear in the GRI G4 Sustainability Guidelines. So, using this order, this study is more readily comparable with the Guidelines. Performance indicators which are showed in this paper have been grouped together by:

Economic indicators: The 'GRI G4 Sustainability Guidelines' recommends 9 economic indicators. All of them apply in a university context. Although it is true that in some cases it is necessary further adjustment to target framework.

Environmental indicators: 'The Guidelines' recommends 34 environmental indicators but, only 30 apply in the University of Cordoba and therefore they can be used in other Spanish universities. Product design, production, marketing and use indicators are not applicable because these are not the main functions of the University.

Social indicators: This is the most difficult category to gather information about it or to apply to university context. 'The Guidelines' recommends 48 social indicators and we only propose 42 adapted indicators. The rest of them concern nonexistent or already rendered obsolete aspects. 


\section{Limitations}

Some limitations of our study should be noted and discussed. Firstly, we acknowledge that we have used a small sample of universities and reports and there is a lack of representativeness of the Spanish university system. Therefore, it is recommended to continue the investigation in order to collect additional information. Secondly, due to the fact that our proposal has not been validated by the opinion of large group of expert, we had to identify what they are the university material aspect and to validate them through the participation of the stakeholders and more expert opinions.

\section{Practical conclusions}

Despite the fact that there is not a sector supplement tailored for universities in order to help them to draw up social responsibility reports according to the Guidelines, this is the most used international standard (in its 2011 version) in Spanish universities. Unfortunately, due to the fact that 'the Guidelines' is not a specific document for higher education institutions, it is difficult to implement it in university context, especially, where the human resources management, product liability and environmental no directly related to the activity performance are concerned.

The result of this study is a useful tool that can help public universities in Spain to overcome the difficulties resulting from the lack of performance indicators in order to develop university social strategies. Our proposal of indicators facilitates that social responsibility reports can adjust to the new reality that the 'GRI G4 Sustainability Guidelines' means.

\section{Original value}

According to the literature, it is necessary to create useful tools that make easier identification of social responsibility indicators according to the material aspects identified by the GRI (economic, environmental and social indicators) within its last version and to the university context. In that sense, it should be pointed out here that the original value of this work is based on giving response to this need, showing a set of university social responsibility performance indicators compliant with the GRI G4 Sustainability Guidelines (the most recent version of this Guidelines).

KEYWORDS: Indicator, Global Reporting Initiative, Social Responsability, University, Sustainability. 


\section{1 .- Introducción}

Aunque el concepto de Responsabilidad Social, desde sus primeras definiciones, ha estado asociado a la actividad empresarial (Vega, 2009), su modelo de gestión es aplicable a instituciones de todo tipo tanto privadas como públicas, incluidas las instituciones de educación superior y universidades. Para las organizaciones privadas, la planificación estratégica basada en la Responsabilidad Social (RS) tiene como finalidad principal mejorar su posición en el mercado a través de la imagen, la reputación o el fortalecimiento de marca (Ganga y Navarrete, 2012). Sin embargo, para las instituciones públicas, el objetivo primordial de la RS es la mejora de la eficiencia, así como los procesos de transparencia y rendición de cuentas (Casani y Pérez-Esparrells, 2009)

En el contexto de la Universidad, la Responsabilidad Social Universitaria (RSU) es definida por Vallaeys (2008, p. 209) como "una política de mejora continua de la Universidad hacia el cumplimiento efectivo de su misión social mediante cuatro procesos: gestión ética y ambiental de la institución; formación de ciudadanos responsables y solidarios; producción y difusión de conocimientos socialmente pertinentes; participación social en promoción de un desarrollo más humano y sostenible". El mismo autor señala que dicho proceso de mejora continua puede ser llevado a cabo a través del autodiagnóstico de la propia institución, el cual se apoya en el uso de indicadores como herramienta de transparencia y de evaluación de resultados (Rodríguez, 2010).

Las evaluaciones basadas en indicadores son de gran utilidad para el proceso de toma de decisiones ya que, en general, son más fácilmente medibles, comparables y objetivas que otros tipos de evaluaciones (Dalal-Clayton y Bass, 2002, citado por Lozano, 2006). Sin embargo, Larrán et al (2012) identifican la falta de indicadores de rendimiento propios de la actividad universitaria como una de las barreras que frenan el desarrollo de estrategias de responsabilidad social en las instituciones de educación superior.

Una reflexión sobre los impactos de la actividad universitaria sobre su entorno y sobre los procesos que constituyen la función social de la Universidad (Vallaeys, de la Cruz y Sasia, 2009) nos lleva a la conclusión de que la legitimidad de las funciones de las instituciones de educación superior viene determinada por los grupos de interés con los que se relacionan (De la Cuesta y Valor, 2003). El compromiso universitario con la sociedad puede manifestarse con distintas acciones según sus prioridades y las de sus grupos de interés (Aldeanueva, 2011). Sin embargo, las diversas actividades que llevan a cabo las universidades y que podrían englobarse en el ámbito de la responsabilidad social, no siempre son identificadas como tal por estos grupos de interés. Uno de los motivos es que se tra- 
tan de iniciativas de carácter trasversal y que afectan a diversas dimensiones universitarias, lo que dificulta su visión de conjunto (Barañano y Rey, 2012).

Algunos mecanismos de comunicación, como las memorias de RSU, ayudan a las universidades a generar una visión integrada de su actividad que materialice su contribución a la sostenibilidad 1 más allá de compromisos y declaraciones de intenciones. De este modo, las universidades socializan su gestión y permiten su verificación por parte de sus grupos de interés (Valarezo y Túñez, 2014). Además, la transparencia en la gestión atrae mayores aportes de financiación y patrocinios, crea reputación y buena imagen, así como reducción de los riesgos por conflictos con los grupos de interés (De la Red, 2009).

Debido a su utilidad y ventajas para las instituciones universitarias, la elaboración de memorias de RSU y el uso de indicadores de medición se ha ido extendiendo en las universidades españolas, pasando de ser una práctica minoritaria (Vallaeys, 2008; Benavides, 2012) a ser un denominador común para más del $60 \%$ de las universidades españolas que tienen integrado la RSU en sus modelos de gestión (Forética, 2016).

Uno de los mejores mecanismos para la rendición de cuentas basados en el enfoque triple bottom line ${ }^{2}$ según Moneva y Marín (2012) y uno de los más usados a nivel nacional (Forética, 2016) es la "Guía para la elaboración de memorias de sostenibilidad" (en adelante, la Guía) propuesta por el Global Reporting Initiative (GRI). El objetivo de la Guía es ayudar a las organizaciones a elaborar memorias de responsabilidad social significativas en las que se recojan datos útiles sobre las cuestiones más importantes para cada organización relacionadas con la sostenibilidad (Global Reporing Initiative [GRI], 2013b). Dicho documento propone una serie de indicadores del desempeño ambiental, económico y social sobre los que las organizaciones pueden trabajar para informar acerca de sus impactos en estos tres ámbitos.

Hasta el momento, se han realizado cinco revisiones de la Guía GRl: la primera, del año 2000, denominada G1, la de 2002 (G2), la de 2006 (G3), la de 2011 (G3.1) y la más reciente, del 2013 (G4). Según la Guía G4, todas las memorias que publicadas después del 31 de diciembre de 2015 deben elaborarse "de conformidad" con esta última versión. Incluso recomienda la versión G4 a aquellas organizaciones que se dispongan a elaborar su primera memoria, aunque no vayan a cumplir los requisitos de las opciones "de conformidad" en los informes.

1.- Conjunto de criterios orientados al comportamiento ético con todo lo que nos rodea (recursos, personas, espacios...), de modo que permita lograr una equidad intra e intergeneracional así como gestionar las relaciones con el medio natural y social, manteniendo su disponibilidad y equilibrio ecológico, y promoviendo una distribución más equitativa y justa de los recursos, beneficios y costes ambientales (Conferencia de Rectores de las Universidades Españolas, 2012).

2.- Se basa en atender las demandas de los distintos grupos de interés a través de la comunicación de resultados económicos, sociales y ambientales de la organización. El concepto fue introducido por John Elkington en Cannibals with Forks (1997) (Orellana, 2006). 
A pesar de que no existe un suplemento de la guía del GRI para la elaboración de memorias de RS en instituciones educativas, éste es el modelo más aplicado por las universidades españolas (Forética, 2016). Si bien es cierto que, al no ser un documento específico para universidades, según Reyes, Montes y Laguna (2014), la Guía resulta de difícil aplicación en el contexto universitario sobre todo en aquellos indicadores vinculados con la gestión de los derechos humanos, con la responsabilidad del producto y con el desempeño ambiental que no están directamente relacionados con la actividad (gestión de residuos, biodiversidad, etc.).

Por tanto, resultan necesarias herramientas que faciliten la identificación de indicadores de RSU acordes con los aspectos materiales identificados por el GRI (económicos, ambientales y sociales) en su última versión de la Guía y centrados en la actividad universitaria. En este sentido, el valor original de este trabajo se basa en dar respuesta a esta necesidad recogiendo una propuesta de indicadores de desempeño de la RSU ajustados a la Guía G4 del GRI, la versión más reciente de este estándar internacional.

Teniendo en cuenta la relevancia del papel de la gestión socialmente responsable en las universidades y de la transparencia en la puesta en práctica de dicha gestión, así como la falta de herramientas estandarizadas para la rendición de cuentas que faciliten la comparabilidad de las iniciativas llevadas a cabo por las instituciones y el análisis de su evolución en el tiempo (Moneva y Martín, 2012), este artículo presenta una propuesta marco para elaborar memorias de RS para universidades públicas, "de conformidad" con la Guía G4 (GRI, 2013a).

El objetivo general del artículo es elaborar una relación de indicadores adaptados a la realidad de las universidades públicas españolas con un enfoque basado en la Guía G4 del GRI (GRI, 2013a). Para ello, se han identificado los siguientes objetivos específicos analizando su aplicación en un estudio de caso:

- Identificar los indicadores universitarios acordes con el GRI de una memoria de RSU.

- Analizar cómo se ajusta la información de esta memoria a los indicadores de desempeño de la Guía G4 (GRI, 2013a)

- Completar el listado de indicadores realizando una serie de propuestas que permita elaborar las siguientes memorias de RSU "de conformidad" a la Guía G4 del GRI (GRI, 2013a). 


\section{2.- Metodología}

En el marco de las universidades públicas españolas y la RSU, se ha realizado un estudio de caso cuyo objeto es la Universidad de Córdoba (UCO) y en el que se analiza la elaboración de sus memorias de RSU para su adaptación a la última versión de la Guía del GRI (GRI, 2013a).

Actualmente, las universidades españolas, para la elaboración de sus memorias de RS optan por los modelos propuestos por el GRI en su versión G3.1 de 2011 o por el Foro de Foro de los Consejos Sociales de las Universidades Públicas Andaluzas; o bien, por una combinación de ambos, como es el caso de la Universidad de Córdoba (Forética, 2016)

Por este motivo, consideramos que los indicadores de desempeño empleados por la UCO pueden ser también de aplicación a otras universidades españolas que deciden elaborar sus memorias de RS siguiendo los mismos modelos.

Para el desarrollo de esta investigación, se ha empleado el estudio de caso como herramienta principal. Según Yin (2009), esta metodología de investigación es particularmente válida cuando pretendemos responder a una pregunta del tipo "cómo", disponiendo de escaso control sobre los hechos estudiados y siendo el tema de actualidad. Aplicando estos criterios de idoneidad, la pregunta de investigación planteada es: ¿Cómo puede la UCO adaptar los indicadores de su memoria de responsabilidad social a la nueva realidad de la Guía G4 publicada por el GRI? En este caso, el investigador no dispone de control sobre los hechos estudiados, pues se trabaja sobre documentos ya publicados. Además, la actualidad del tema viene dada por la fecha de publicación de la última versión de la Guía del GRI (mayo de 2013).

Según Hart (2013), citando a Berg (2007), Glesne (2006) y Yin (1989), el estudio de caso individual es reconocido como una oportunidad de aprendizaje válida con la habilidad de informar y, en algunos casos, generar teoría.

Se ha elegido la UCO como objeto del estudio de caso por diversos motivos:

- La UCO ha publicado sus cuatro últimas memorias anuales siguiendo la propuesta del Foro de Consejos Sociales de las Universidades Públicas Andaluzas (Foro de Consejos Sociales de las Universidades Públicas Andaluzas [FCCSSA], 2009). En sus informes para los cursos 2011/2012, 2013-2013 y 2013/2014, la UCO autocalifica el texto siguiendo los requisitos del GRI (en su versión 3.1) y comunica su correspondiente listado de indicadores (Forética, 2016).

- Desde abril de 2015, la UCO se encuentra inmersa en el proceso de elaboración de su II Plan Estratégico en el que queda patente, desde sus inicios, la importancia de la función social de la institución y su gestión socialmente responsable. 
- La propia institución demanda el desarrollo herramientas que faciliten la identificación de indicadores de desempeño para elaborar sus memorias de RS "de conformidad" con la Guía del GRI, por lo que el presente trabajo tiene un aplicación práctica directa, permitiendo así su ensayo, análisis y mejora.

- Existe un conocimiento previo del proceso de elaboración de las memorias de RS de la UCO al haber podido participar en su redacción.

Al igual que en los estudios de De Gobrois (2012); Moneva y Llena (1996); Mustata, Bonaci, Hintea y Neamtu (2013) y Toppinen (2013), el presente trabajo se basa en el análisis de la información contenida en documentos públicos y accesibles a través de la web de las organizaciones, como informes anuales, memorias de RS u otro tipo de memorias.

Tras el estudio comparativo de las Guías G3.1 (GRI, 2011a) y G4 del GRI (GRI, 2013a) y el "Resumen de cambios indicadores desde G3.1 a las directrices G4" (GRI, 2013c), el siguiente paso, ya centrado en el caso de la UCO, fue el análisis del contenido de su memoria de RS para el curso 2011/2012 (UCO, 2013).

En este punto se identificaron los indicadores de desempeño que se podían ajustar, en mayor 0 menor grado, a la propuesta de indicadores de la Guía G4 del GRI.

Con el objetivo de poder identificar los indicadores más frecuentes en el contexto de las universidades públicas españolas y siguiendo la propuesta de Villarreal y Landeta (2010), se emplearon múltiples fuentes documentales para elaborar nuestra propuesta (ver Anexo I)

Una vez recopilados los indicadores empleados por la UCO y el resto de universidades de la muestra para cada uno de los indicadores de desempeño de la Guía G3.1 (GRI, 2011a), la siguiente tarea fue hacer una selección de los mismos para elaborar nuestra propuesta, siguiendo los criterios que se citan a continuación:

- Indicadores empleados por la propia UCO.

- Ajuste del indicador a los requisitos de la Guía G4 del GRI.

- Mayor frecuencia de uso del indicador en las universidades de la muestra.

A continuación, el contenido de cada indicador fue revisado y adaptado a la Guía G4 (GRI, 2013a), identificando, para cada propuesta, su función y justificación.

El paso final fue la verificación de las propuestas a través de la opinión de experto, contando con la colaboración de la máxima responsable en la elaboración de las memorias de RS de la UCO en 2013, que ocupaba el cargo de la Secretaría General de la universidad. Con su colaboración, se pudo comprobar de forma cualitativa la pertinencia, comprensibilidad y fiabilidad (Naciones Unidas, 2008) de los indicadores propuestos. 


\section{3.- Resultados}

Como resultado de esta investigación se ha obtenido una propuesta de indicadores que permite a la Universidad de Córdoba y al resto de las universidades públicas españolas elaborar una memoria de RSU conforme a la Guía G4 (GRI, 2013a). De igual modo, considerando el carácter internacional de la Guía G4 en la cual se basa el actual trabajo, la propuesta en él presentada podría aplicarse a otras instituciones de educación superior de carácter internacional.

Dicha propuesta se ha dividido en tres apartados según se trata de indicadores económicos, ambientales o sociales. A su vez, dentro de cada apartado, los indicadores se agrupan por "aspectos" en el mismo orden que aparece en la Guía G4. Con este orden se pretende que el presente trabajo sea más fácilmente comparable con este estándar internacional.

La nota explicativa relacionada con cada indicador (enumeradas al final del artículo de la i a la vi) hace referencia a la justificación que nos ha llevado a hacer dicha propuesta.

Para entender el conjunto de los resultados y las diferencias con respecto a los indicadores recogidos en la Guía G4, hay que tener en cuenta que el objetivo de la administración pública (en nuestro caso, la Universidad) es alcanzar un fin social mediante el empleo de unos recursos dados. Este objetivo no se alcanza mediante la producción y distribución de bienes y/o servicios, tal como sucede en el sector privado (Samaniego, 1998). Si bien se considera que el desempeño de las actividades de docencia, investigación y gestión que UCO se puede medir y evaluar a través de indicadores asociados a servicios.

\section{a) Indicadores económicos}

Los 9 indicadores económicos propuestos en la Guía G4 (GRI, 2013a) resultan de aplicación a la actividad universitaria de la UCO, aunque sobre cinco de ellos, esta universidad no ha comunicado información hasta ahora.

\section{Desempeño económico}

En la Tabla 1 se muestran los indicadores relacionados con el desempeño económico y las propuestas asociadas. 
Para el indicador G4-EC2, la perspectiva en la identificación de los riesgos y oportunidades relacionados con el cambio climático difiere entre una empresa o industria y la universidad, la cual no se centra tanto en los costos de producción, la competitividad o la aparición de nuevos mercados (GRI, 2013a), sino más bien en los costes de gestión y la transferencia de conocimiento.

Por otro lado, el indicador G4-EC4, en el contexto de la universidad pública, no trata de medir la contribución de los gobiernos a la institución, que se supone mayoritaria (Escardíbul y Pérez, 2013). Más bien, el objetivo es el contrario: identificar la presencia de financiación privada en la universidad pública para así evaluar su pertinencia.

\section{Tabla 1. Indicadores sobre desempeño económico}

\begin{tabular}{|l|l|}
\hline CÓDIGO G4 & PROPUESTAS DE INDICADORES UNIVERSITARIOS \\
\hline & $\begin{array}{l}\text { - Descripción e importe (en miles de } € \text { ) de gastos e ingresos presupuestados y liquidados en } 2011 . \\
\text { - Límite autorizado de endeudamiento (en millones de } € \text { ). } \\
\text { - Nivel de endeudamiento (en millones de } € \text { ). }\end{array}$ \\
& $\begin{array}{l}\text { - Origen e importe (en \% y en miles de } € \text { ) de la financiación en el presupuesto liquidado de } 2011 . \\
\text { - Descripción e importe (en \% y en miles de } € \text { ) de la previsión de ingresos y gastos para } 2012 . \\
\text { - Descripción y evolución del importe (en } € \text { y \% con respecto al curso anterior) de inversiones realizadas } \\
\text { en infraestructuras y equipamiento. } \\
\text { - Descripción e importe (en } € \text { ) de servicios ejecutados o adquiridos en el curso 2011-12. } \\
\text { - Evolución del importe total (en } € \text { ) destinado a investigación y porcentaje en relación al total del presupuesto. } \\
\text { - Evolución del importe (en } € \text { ) destinado a becas y ayudas. }\end{array}$ \\
\hline G4-EC2 & $\begin{array}{l}\text { Relación de las oportunidades (como la investigación en procesos bajos en carbono o la relación del cambio } \\
\text { climático sobre diversos aspectos de la sociedad y la economía) y los riesgos (como el incremento del } \\
\text { consumo energético o la menor disponibilidad de recursos hídricos) que el cambio climático ejerce sobre } \\
\text { la actividad universitaria. }\end{array}$ \\
\hline G4-EC3 ${ }^{\text {ii }}$ & $\begin{array}{l}\text { - Descripción de medidas para fomentar la conciliación de la vida familiar y laboral. } \\
\text { - Evolución de los fondos destinados a Acción Social (en } € \text { ). } \\
\text { - Evolución del importe de los incentivos a la jubilación anticipada (en } € \text { ). }\end{array}$ \\
\hline G4-EC4 & \begin{tabular}{l} 
Origen de la financiación en el presupuesto liquidado (en miles de $€$ y en \%) \\
\hline
\end{tabular}
\end{tabular}

i) La UCO informa sobre el indicador, el cual, no experimenta cambios de contenido en su transición de la Guía 3.1 (GRI, 2011a) a la versión G4 (GRI, 2013a).

ii) La UCO no comunica, por el momento, información relativa a este indicador.

\section{Presencia en el mercado}

El indicador relativo a los salarios pone de manifiesto el bienestar de los empleados, aunque en el caso de las universidades públicas dichas retribuciones vienen determinadas por la legislación vigente. 


\section{Tabla 2. Indicadores económicos sobre presencia en el mercado}

\begin{tabular}{|l|l|}
\hline CÓDIGO G4 & PROPUESTAS DE INDICADORES UNIVERSITARIOS \\
\hline G4-EC5 ${ }^{\text {i }}$ & $\begin{array}{l}\text { - Sueldo base anual (en } € \text { ) segregado por colectivo y categoría profesional. } \\
\text { - Relación del sueldo base expresado en \% con respecto al salario bruto mínimo interprofesional de cada año. }\end{array}$ \\
\hline G4-EC6 6 ii & Proporción de puestos de dirección nacidos en la provincia. \\
\hline
\end{tabular}

ii) La UCO no comunica, por el momento, información relativa a este indicador.

Consecuencias económicas indirectas

En el contexto de las universidades, el impacto económico indirecto de mayor relevancia sobre la sociedad es el relacionado con la transferencia del conocimiento y de la actividad investigadora.

\section{Tabla 3. Indicadores económicos sobre consecuencias económicas indirectas}

\begin{tabular}{|l|l|}
\hline CÓDIGO G4 & PROPUESTAS DE INDICADORES UNIVERSITARIOS \\
\hline \multirow{3}{*}{ G4-EC7 iii } & $\begin{array}{l}\text { - Evolución del importe y descripción de inversiones realizadas en infraestructuras. } \\
\text { - Tipo de usuario de las infraestructuras (público en general, estudiantes, trabajadores). } \\
\text { - No de usuarios al año. } \\
\text { - Tipo de gestión de las infraestructuras (propia o externa). }\end{array}$ \\
\hline G4-EC8 ii & $\begin{array}{l}\text { - Valor medio de la investigación contratada (en } € \text { ). } \\
\text { - Número y valor (en } € \text { ) de proyectos anuales desarrollados para administraciones públicas, para empresas de entidades. } \\
\text { - Valor medio (en } € \text { ) de servicios técnicos y asesoría realizados por la universidad. } \\
\text { - Indicadores de transferencia de resultados: media anual de solicitudes de patentes, número de licencias } \\
\text { e ingresos que generan. }\end{array}$ \\
\hline
\end{tabular}

ii) La UCO no comunica, por el momento, información relativa a este indicador.

iii) La UCO informa sobre este indicador aunque no lo identifica como tal.

\section{Prácticas de adquisición}

El indicador G4-ECgii está relacionado con la cadena de suministro y considera proveedores tanto de productos como de servicios. Los ítems propuestos para este indicador son:

- Relación del importe de los contratos a proveedores de la provincia (en \%) con respecto al importe total de todos los contratos adjudicados en el periodo de la memoria.

- Relación el importe de las facturas abonadas a proveedores de la provincia (en \%) con respecto al total abonado a todas las facturas abonadas en el periodo de la memoria. 


\section{b) Indicadores ambientales}

La UCO dispone de información en su memoria RSU para el curso 2011/2012 (UCO, 2013) de información relacionada con el $40 \%$ de indicadores de desempeño ambiental propuestos por la Guía G4 (GRI, 2013a) y que son de aplicación a la actividad universitaria. En líneas generales, resultan aplicables los indicadores asociados a servicios y que se relacionan con aspectos ambientales de la gestión, la docencia o la investigación. En cambio, no son aplicables los indicadores relacionados con productos (diseño, producción, comercialización y uso). En cifras, son de aplicación 30 de los 34 indicadores ambientales de la Guía G4.

Materiales y Energía

\section{Tabla 4. Indicadores ambientales sobre materiales}

\begin{tabular}{|l|l|}
\hline CÓDIGO G4 & PROPUESTAS DE INDICADORES UNIVERSITARIOS \\
\hline G4-EN1 ${ }^{\text {iii }}$ & $\begin{array}{l}-\mathrm{N}^{\circ} \text { de paquetes de } 500 \text { hojas de papel adquiridos. } \\
-\mathrm{N}^{\circ} \text { de tóneres y cartuchos de tinta adquiridos. }\end{array}$ \\
\hline \multirow{3}{*}{ G4-EN2 2 iii } & $\begin{array}{l}-\mathrm{N}^{\circ} \text { de paquetes de } 500 \text { hojas de papel reciclado con respecto al número total de paquetes de } 500 \text { hojas } \\
\text { de papel blanco y reciclado adquiridos (expresado en \%). } \\
-\mathrm{N}^{0} \text { de tóneres y cartuchos de tinta reciclados con respecto al total de tóneres y cartuchos de tinta } \\
\text { adquiridos (expresado en \%). }\end{array}$ \\
\hline
\end{tabular}

iii) La UCO informa sobre este indicador aunque no lo identifica como tal.

\section{Tabla 5. Indicadores ambientales sobre consumo y generación de energía}

\begin{tabular}{|l|l|}
\hline CÓDIGO G4 & PROPUESTAS DE INDICADORES UNIVERSITARIOS \\
\hline G4-EN3 & $\begin{array}{l}\text { Consumo energético total = combustible no renovable (gas natural, gasóleo, propano) + combustible } \\
\text { renovable (biomasa, biodiesel) + electricidad suministrada por comercializadora + electricidad autogenerada } \\
\text { para autoconsumo (cogeneración) - electricidad vendida. }\end{array}$ \\
\hline G4-EN5V & $\begin{array}{l}\text { Valor del indicador G4-EN3 relativizado por número de usuarios (trabajadores + alumnos), por } \mathrm{m}^{2} \mathrm{de} \\
\text { superficie o por días lectivos. }\end{array}$ \\
\hline G4-EN4 & No se hacen propuestas. El indicador no es de aplicación al no ser la actividad universitaria un proceso productivo. \\
\hline G4-EN6 & $\begin{array}{l}\text { Ahorro energético (electricidad y gas natural) en } € \text { en kilowatios-hora y en } \mathrm{m}^{3} \text { debido a las iniciativas puestas } \\
\text { en marcha para reducir el consumo o hacerlo más eficiente (por ejemplo, cierre de las instalaciones en } \\
\text { periodos vacacionales). }\end{array}$ \\
\hline G4-EN7ii & $\begin{array}{l}\text { Variación (en \%) del consumo energético entre dos periodos (curso lectivo o año natural) del indicador } \\
\text { G4-EN3 relativizado por días lectivos. }\end{array}$ \\
\hline
\end{tabular}

i) La UCO informa sobre el indicador, el cual, no experimenta cambios de contenido en su transición de la Guía 3.1 (GRI, 2011a) a la versión G4 (GRI, 2013a). ii) La UCO no comunica, por el momento, información relativa a este indicador. iv) La UCO informa sobre este indicador, el cual experimenta ciertos cambios en su transición de la versión G3.1 (GRI, 2011a) a la versión G4 (GRI, 2013a).

v) Indicador nuevo en la Guía G4 (GRI, 2013a) que no está presente en la versión G3.1 (GRI, 2011a). 
Agua

\section{Tabla 6. Indicadores ambientales sobre consumo de agua}

\begin{tabular}{|l|l|}
\hline CÓDIGO G4 & PROPUESTAS DE INDICADORES UNIVERSITARIOS \\
\hline \multirow{2}{*}{ G4-EN8 } & $\begin{array}{l}\text { - Volumen de agua de consumo }\left(\text { en } \mathrm{m}^{3} \text { ). }\right. \\
\text { - Tasa de variación (en \%) del consumo de agua entre dos periodos (curso lectivo o año natural) } \\
\text { - Origen de las fuentes de suministro (aguas superficiales, subterráneas, pluviales, suministro de agua } \\
\text { municipal, etc.) }\end{array}$ \\
\hline \multirow{2}{*}{ G4-ENgiii } & $\begin{array}{l}\text { - Información relativa a cada fuente de agua: } \\
\text { - Consumo (en } \mathrm{m}^{3} \text { ). } \\
\text { - Tamaño de la fuente. } \\
\text { - Figura de protección (si la tiene). } \\
\text { - Importancia para la comunidad local, indicando si existen otros usuarios además de la universidad. }\end{array}$ \\
\hline G4-EN10 ${ }^{\text {iii }}$ & $\begin{array}{l}\text { Existencia o ausencia de sistemas de recogida de aguas pluviales para su uso como riego, limpieza, agua para } \\
\text { cisternas o bocas de incendios o sistemas de recuperación de aguas grises en los edificios; así como la } \\
\text { capacidad de los mismos, en su caso. }\end{array}$ \\
\hline
\end{tabular}

i) La UCO informa sobre el indicador, el cual, no experimenta cambios de contenido en su transición de la Guía 3.1 (GRI, 2011a) a la versión $\mathrm{G} 4$ (GRI, 2013a)

iii) La UCO informa sobre este indicador aunque no lo identifica como tal.

\section{Biodiversidad}

\section{Tabla 7. Indicadores ambientales sobre biodiversidad}

\begin{tabular}{|l|l|}
\hline CÓDIGO G4 & PROPUESTAS DE INDICADORES UNIVERSITARIOS \\
\hline G4-EN11 ${ }^{\text {i }}$ & $\begin{array}{l}\text { Descripción de espacios verdes en el interior de los campus, jardines y huertos y listado de especies que } \\
\text { habitan en ellos } 3\end{array}$ \\
\hline G4-EN12 & $\begin{array}{l}\text { Relación de los impactos negativos (ruido, iluminación nocturna, tráfico rodado) y positivos (conservación y } \\
\text { mejora de espacios verdes) generados por la actividad diaria en las áreas descritas en el indicador G4-EN11. }\end{array}$ \\
\hline G4-EN13ii & $\begin{array}{l}\text { Descripción de acciones de voluntariado ambiental relacionadas con la restauración o protección de hábitats } \\
\text { (p. ej., reforestaciones) }\end{array}$ \\
\hline G4-EN14ii & $\begin{array}{l}\text { Inventario de especies que habitan en los campus e identificación de las que se encuentran en la lista roja } \\
\text { de la UICN4 o en el Listado de Especies Silvestres en Régimen de Protección Especial y del Catálogo Español } \\
\text { de Especies Amenazadas (Real Decreto 139/2011, de 4 de febrero). }\end{array}$ \\
\hline
\end{tabular}

ii) La UCO no comunica, por el momento, información relativa a este indicador.

3.- Un ejemplo interesante es la publicación de la propia UCO sobre las especies que habitan el Campus de Rabanales, disponible en http://www.uco.es/servicios/dgppa/images/sepa/101especies.pdf

4.- Unión Internacional para la Conservación de la Naturaleza, cuya misión es influir, estimular y apoyar a las sociedades de todo el planeta, con objeto de mantener la integridad de la naturaleza y asegurar el uso equitativo y ecológicamente sostenible de los recursos naturales.

CIRIEC-España, Revista de Economía Pública, Social y Cooperativa

ISSN: 0213-8093 


\section{Emisiones}

\section{Tabla 8. Indicadores ambientales sobre emisiones}

\begin{tabular}{|c|c|}
\hline CÓDIGO G4 & PROPUESTAS DE INDICADORES UNIVERSITARIOS \\
\hline G4-EN15ii & $\begin{array}{l}\text { Toneladas de } \mathrm{CO}_{2} \text {-eq. del consumo de gas o gasóleo en calderas para calefacción y del transporte de } \\
\text { vehículos propios y de trabajadores. }\end{array}$ \\
\hline G4-EN16ii & Toneladas de $\mathrm{CO}_{2}$-eq. del consumo eléctrico considerando el mix eléctrico español. \\
\hline G4-EN17il & Toneladas de $\mathrm{CO}_{2}$-eq. del transporte del alumnado y de viajes institucionales. \\
\hline G4-EN18V & $\begin{array}{l}\text { - Suma de los valores de G4-EN15 y G4-EN16 relativizado por } \mathrm{m}^{2}, \mathrm{n}^{0} \text { de alumnos } \mathrm{y} / 0 \mathrm{n}^{0} \text { de empleados. } \\
\text { - Valor de G4-EN15 entre } \mathrm{m}^{2}, \mathrm{n}^{0} \text { de alumnos y/o } \mathrm{n}^{0} \text { de empleados. } \\
\text { - Valor de G4-EN16 entre } \mathrm{m}^{2}, \mathrm{n}^{0} \text { de alumnos y/o } \mathrm{n}^{\circ} \text { de empleados. } \\
\text { - Valor de G4-EN17 entre } \mathrm{m}^{2}, \mathrm{n}^{0} \text { de alumnos y/o } \mathrm{n}^{\circ} \text { de empleados. }\end{array}$ \\
\hline G4-EN19ii & $\begin{array}{l}\text { - Iniciativas que han logrado una disminución de las emisiones. } \\
\text { - Toneladas de } \mathrm{CO}_{2} \text {-eq. reducidas en relación a las iniciativas descritas. }\end{array}$ \\
\hline G4-EN20 & $\begin{array}{l}\text { No se hacen propuestas. No se considera de aplicación ya que la actividad de la UCO no es su susceptible } \\
\text { de emitir sustancias que agotan la capa de ozono }{ }^{5} \text {. }\end{array}$ \\
\hline G4-EN21ii & $\begin{array}{l}\text { - Emisiones debidas al consumo de combustibles (en toneladas): } \mathrm{SO}_{\chi}, \mathrm{NO}_{\chi}, \text { compuestos orgánicos } \\
\text { volátiles no metano (COVNM), } \mathrm{CO} \text {, partículas suspendidas, } \mathrm{Hg} \text { (este último en } \mathrm{Kg} \text {.) } \\
\text { - Emisiones debidas al consumo eléctrico según el mix eléctrico español (en toneladas): } \mathrm{SO}_{X}, \mathrm{NO}_{X}, \mathrm{COVNM} \text {, } \\
\mathrm{CH}_{4}, \mathrm{CO} \text {, metales pesados (en } \mathrm{Kg} \text {.) }\end{array}$ \\
\hline
\end{tabular}

ii) La UCO no comunica, por el momento, información relativa a este indicador.

v) Indicador nuevo en la Guía G4 (GRI, 2013a) que no está presente en la versión G3.1 (GRI, 2011a).

\section{Efluentes y residuos}

La evolución en la generación de los residuos puede poner de manifiesto los esfuerzos por mejorar las infraestructuras y la gestión para una recogida selectiva, así como la eficacia de las actividades de sensibilización hacia la comunidad universitaria. 


\section{Tabla 9. Indicadores ambientales sobre efluentes y residuos}

\begin{tabular}{|l|l|}
\hline CÓDIGO G4 & PROPUESTAS DE INDICADORES UNIVERSITARIOS \\
\hline G4-EN22 & $\begin{array}{l}\text { - Tipo de vertido (aguas residuales urbanas) y destino del mismo (estación depuradora). } \\
\text { - Volumen total de aguas residuales urbanas, pudiéndose asumir el dato de agua de consumo como } \\
\text { agua de vertido. }\end{array}$ \\
\hline G4-EN23 & $\begin{array}{l}\text { - Kg. de los distintos tipos de residuos: residuos peligrosos, residuos de aparatos eléctricos y electrónicos, } \\
\text { pilas, tóner, etc. } \\
\text { - Destino de cada tipo de residuo (gestor autorizado, planta de tratamiento, vertedero, etc.) }\end{array}$ \\
\hline G4-EN24il & $\begin{array}{l}N^{\circ} \text { de derrames (significativos o no) que se registran en servicios o departamentos con instalaciones } \\
\text { provistas de depósitos o instalaciones de almacenamiento de productos químicos o líquidos peligrosos. }\end{array}$ \\
\hline G4-EN25 & $\begin{array}{l}\text { Destino final (valorización, reciclado o eliminación según Ley 22/2011, de 28 de julio, de residuos y suelos } \\
\text { contaminados) y peso de cada uno de los tipos de residuos peligrosos generados. }\end{array}$ \\
\hline G4-EN26 & No se hacen propuestas al no ser aplicable por la naturaleza de los vertidos de la UCO. \\
\hline
\end{tabular}

i) La UCO informa sobre el indicador, el cual, no experimenta cambios de contenido en su transición de la Guía 3.1 (GRI, 2011a) a la versión G4 (GRI, 2013a)

ii) La UCO no comunica, por el momento, información relativa a este indicador.

\section{Productos y servicios}

Los indicadores de la Guía G4 sobre aspectos ambientales relacionados con productos, no son de aplicación al contexto universitario por ser otra su actividad principal. Este es el caso de los indicadores G4-EN4 y G4-EN28 para los cuales no se hacen propuestas. Sin embargo, sí es de aplicación el indicador de aspectos ambientales asociados a servicios, el G4-EN27', que se concreta en los siguientes ítems:

Descripción y cuantificación ( $\mathrm{n}^{\circ}$ de actividades y $\mathrm{n}^{\circ}$ de participantes) de iniciativas relacionadas con:

- Formación y sensibilización ambiental

- Gestión eficiente de recursos (agua, energía, papel) y consumo responsable.

- Fomento de la movilidad sostenible.

- Sistemas de gestión ambiental certificados.

El indicador G4-EN27 propuesto informa de una manera cualitativa (no cuantitativa, como propone la Guía G4) sobre cómo se mitiga el impacto ambiental de la actividad universitaria a través de la sensibilización de alumnos, trabajadores y contratas. 


\section{Cumplimiento regulatorio ambiental}

El nivel de incumplimiento de la legislación ambiental indica el grado de compromiso de la universidad y su equipo directivo para asegurar que su actividad se desarrolla se acorde con ciertos parámetros de desempeño (GRI, 2013a). Esta información se recoge en el indicador G4-EN29 a través de los siguientes ítems:

- $\quad N^{0}$ e importe (en $€$ ) de multas o sanciones por incumplimiento de normativa ambiental.

- $\quad N^{0}$ de multas no monetarias por incumplimiento de normativa ambiental. Si no existen, informar con una breve declaración.

\section{Transporte}

En 2013, la UCO informa sobre su huella de carbono ${ }^{6}$, las actividades de fomento de la movilidad sostenible o el reparto modal de estudiantes según el transporte utilizado, pero no informa del impacto ambiental del transporte de personal (en el que se puede incluir a los estudiantes). La propuesta que se hace para el indicador G4-EN30Vi es:

- Emisiones del transporte descritos en los indicadores G4-EN17 y G4-EN15.

- Estudios de ruido por el tráfico rodado en campus.

- Huella de carbono (toneladas de $\mathrm{CO}_{2}$ )

\section{General}

El indicador G4-EN31 1 ii para la UCO desglosa el coste económico de limpieza de derrames descritos en G4-EN24 y el de mantenimiento y revisión de instalaciones con emisiones: calderas, vehículos oficiales, etc. También recoge información sobre los gastos e inversiones ambientales en:

- Gestión por cada tipo de residuo.

- Auditorías y certificaciones externas de gestión ambiental.

- Actuaciones de formación y sensibilización ambiental.

- Personal con funciones en gestión, formación y sensibilización ambiental. 


\section{Evaluación ambiental a proveedores}

Estos indicadores son nuevos en la versión G4 de la Guía, informando así sobre el porcentaje de proveedores que se ha seleccionado a partir de la debida diligencia ambiental ${ }^{7}$ y sobre la medida en que se es consciente de los impactos ambientales negativos de la cadena de suministro (GRI, 2013a).

\section{Tabla 10. Indicadores sobre evaluación ambiental a proveedores}

\begin{tabular}{|l|l|}
\hline CÓDIGO G4 & PROPUESTAS DE INDICADORES UNIVERSITARIOS \\
\hline G4-EN32 & $\begin{array}{l}\mathrm{N}^{\circ} \text { de contratos de productos, obras o servicios en cuyas prescripciones técnicas se incluyeron criterios } \\
\text { ambientales. }\end{array}$ \\
\hline & $\begin{array}{l}-\mathrm{N}^{\circ} \text { de empresas evaluadas ambientalmente o } \mathrm{n}^{\circ} \text { de informes ambientales de empresas. } \\
\text { - Relación de aspectos ambientales y magnitud correspondiente por tipo de empresa (consumo de recursos, } \\
\text { cantidad de residuos generados) } \\
-\mathrm{N}^{\circ} \text { de proveedores con los que se han puesto en marcha acciones para minimizar impactos después de } \\
\text { la evaluación. } \\
-\mathrm{N}^{\circ} \text { de proveedores con los que se ha finalizado relación contractual después de la evaluación. }\end{array}$ \\
\hline
\end{tabular}

Mecanismos de reclamación ambiental

El indicador G4-EN34 hace referencia a la eficacia de los mecanismos de reclamación ambientales puestos en marcha. Para la UCO, se concreta en los siguientes puntos:

- $\quad \mathrm{N}^{0}$ de reclamaciones recibidas por temas ambientales y \% de reclamaciones resueltas.

- Mecanismos formales de reclamación.

\section{c) Indicadores sociales}

El ámbito social es el de mayor dificultad a la hora de disponer de información o aplicar los indicadores de la Guía G4. La UCO, en su memoria 2011/2012 (UCO, 2013) informa de menos del 30\% de los indicadores de desempeño social que son de aplicación (28,5\%). Este porcentaje disminuye hasta el 25 si consideramos todos los indicadores propuestos por la Guía (GRI, 2013a). De los 48 indicadores del desempeño social que propone la Guía G4, 42 son de aplicación a la actividad de la UCO.

7.- Integración de los riesgos ambientales previamente al inicio de una relación contractual. Puede lograrse mediante inclusión de cláusulas ambientales en los contratos. También puede darse durante la vigencia del contrato o una vez finalizado (Perea-Sarachaga, 1995). 
Los indicadores sociales se dividen en cuatro apartados relacionados con las prácticas laborales y el trabajo digno, los derechos humanos, la sociedad y la responsabilidad sobre los productos. Este último apartado también recoge indicadores relativos a servicios.

\section{c.1.) Prácticas laborales y trabajo digno}

Indicadores sobre empleo

Estos indicadores informan de la naturaleza de los criterios de contratación en la universidad, así como del grado de bienestar de sus empleados.

\section{Tabla 11. Indicadores sociales sobre empleo}

\begin{tabular}{|c|c|}
\hline CODIGO G4 & ROPUESTAS DE INDICADORES UNIVERSITARIOS \\
\hline G4-LA1Vi & $\begin{array}{l}N^{0} \text { de nuevas incorporaciones para el personal de administración y servicios (PAS), el personal docente e } \\
\text { investigador (PDI), becas y contratos, desglosados por sexo y grupos de edad } 8 \text {. }\end{array}$ \\
\hline G4-LA2 & Programas sociales referenciados en el indicador G4-EC3 ${ }^{9}$. \\
\hline G4-LA3ii & $\begin{array}{l}\text { - No de empleados, desglosados por sexo, que tuvieron derecho a una baja por maternidad o paternidad. } \\
\text { - No de empleados, desglosados por sexo, que ejercieron su derecho a baja por maternidad o paternidad. } \\
\text { - No de empleados que se reincorporaron al trabajo después de que finalizase su baja por maternidad o } \\
\text { paternidad, desglosados por sexo. } \\
\text { - № de empleados que se reincorporaron al trabajo después de que finalizase su baja por maternidad o } \\
\text { paternidad y conservaron su empleo pasados doce meses desde su reincorporación, desglosados por sexo. } \\
\text { - Índice de reincorporación al trabajo. } \\
\text { - Índice de retención. }\end{array}$ \\
\hline
\end{tabular}

i) La UCO informa sobre el indicador, el cual, no experimenta cambios de contenido en su transición de la Guía 3.1 (GRI, 2011a) a la versión $\mathrm{G} 4$ (GRI, 2013a)

ii) La UCO no comunica, por el momento, información relativa a este indicador.

iv) La UCO informa sobre este indicador, el cual experimenta ciertos cambios en su transición de la versión G3.1 (GRI, 2011a) a la versión G4 (GRI, 2013a).

\section{Relaciones entre los trabajadores y la dirección}

A través del indicador G4-LA4ii, se informa de las normas que regulan las relaciones entre los trabajadores y el equipo directivo de la universidad, como indicador indirecto del grado de satisfacción y motivación de los empleados. Este indicador se desglosa en los siguientes ítems:

8.- Se pueden tomar como referencia los grupos etarios de la Guía G4 (menores de 30 años, entre 30 y 50 y mayores de 50) o del Ministerio de Educación (menores de 30 años, de 30 a 49, de 50 a 59 y de 60 años o más) (Ministerio de Educación, Ciencia y Deporte, 2012).

9.- Se debe informar del alcance del reglamento de Acción Social de la UCO. 
- Descripción de las tramitaciones internas de las modificaciones organizativas.

- Referencia a la legislación que regula el acuerdo de las modificaciones sustanciales de las condiciones de trabajo para los empleados públicos (Ley 7/2007, de 12 de abril, del Estatuto Básico del Empleado Público, y IV Convenio Colectivo de las Administraciones Públicas de Andalucía en su artículo 13).

- Convenios colectivos en los que se especifica el periodo de preaviso de cambios operativos u organizativos significativos y las disposiciones relativas a posibles consultas y negociaciones.

Salud y seguridad en el trabajo

\section{Tabla 12. Indicadores sociales sobre salud y seguridad en el trabajo}

\begin{tabular}{|c|c|}
\hline CÓDIGO G4 & PROPUESTAS DE INDICADORES UNIVERSITARIOS \\
\hline G4-LA5ii & $\begin{array}{l}\text { - Número total de miembros y composición de los comités de seguridad y salud. } \\
\text { - \% de la plantilla que forman parte de los comités de seguridad y salud. } \\
\text { - \% de la plantilla a la que se representa en dichos comités. }\end{array}$ \\
\hline G4-LA6Vi & $\begin{array}{l}\text { - No de casos de enfermedades profesionales. } \\
\text { - Indicadores de absentismo, accidentes y siniestralidad, segregados por sexo. }\end{array}$ \\
\hline G4-LA $7^{\text {iV }}$ & $\begin{array}{l}N^{\circ} \text { de trabajadores que desarrollan actividades profesionales con incidencia o riesgo elevado de } \\
\text { determinadas enfermedades. }\end{array}$ \\
\hline G4-LA8 $8^{\mathrm{ii}}$ & $\begin{array}{l}\text { - № de inspecciones periódicas de puestos de trabajo en materia de seguridad y salud. } \\
\text { - Listado de convenios o documentos en los que se ponen de manifiesto acuerdos relacionados con } \\
\text { seguridad y salud (facilitando enlaces a los documentos) } \\
\text { - Mecanismos de reclamación de los trabajadores en materia de seguridad y salud. } \\
\text { - Estructuras para la resolución de problemas en esta materia. } \\
\text { - Programas de formación y educación en prevención de riesgos laborales. } \\
\text { - № de solicitudes de equipos de protección individual. }\end{array}$ \\
\hline
\end{tabular}

ii) La UCO no comunica, por el momento, información relativa a este indicador.

iv) La UCO informa sobre este indicador, el cual experimenta ciertos cambios en su transición de la versión G3.1 (GRI, 2011a) a la versión G4 (GRI, 2013a).

vi) La UCO informa sobre este indicador, el cual no experimenta cambios en su paso a la versión G4 de la Guía, aunque la información ofrecida por la universidad no se ajusta del todo a los requisitos del GRI.

\section{Capacitación y educación}

La formación y los programas de gestión de competencias ayudan a mantener y mejorar el capital humano, así como evaluar el rendimiento de los trabajadores respecto a objetivos comunes, ayudando al desarrollo personal de los individuos (GRI, 2013a). 


\section{Tabla 13. Indicadores sociales sobre capacitación y educación}

\begin{tabular}{|l|l|}
\hline CÓDIGO G4 & PROPUESTAS DE INDICADORES UNIVERSITARIOS \\
\hline G4-LA9Vi & - Promedio de horas de formación por empleada, por empleado varón y por categoría laboral para PAS y PDI. \\
\hline G4-LA10iii & $\begin{array}{l}\text { - Importe (en } € \text { ) destinado a incentivos a la jubilación anticipada. } \\
\text { - Número de excedencias. } \\
\text { - Programas de formación del personal (para PAS y PDI). } \\
\text { - Indemnizaciones por despido. } \\
\text { - Servicios de colocación. }\end{array}$ \\
\hline G4-LA111ii & $\begin{array}{l}\text { - Mecanismos de evaluación de la actividad docente y } \mathrm{n}^{\circ} \text { de evaluaciones realizadas. } \\
\text { - Mecanismos de evaluación de la calidad de los servicios y } \mathrm{n}^{\circ} \text { de evaluaciones realizadas. }\end{array}$ \\
\hline
\end{tabular}

iii) La UCO informa sobre este indicador aunque no lo identifica como tal.

vi) La UCO informa sobre este indicador, el cual no experimenta cambios en su paso a la versión G4 de la Guía, aunque la información ofrecida por la universidad no se ajusta del todo a los requisitos del GRI.

Diversidad e igualdad de oportunidades

La información contenida en el indicador G4-L12 ${ }^{\mathrm{Vi}}$ ofrece una perspectiva del capital humano y permite la comparación entre la diversidad en la base del colectivo de trabajadores y la diversidad del los órganos de gobierno, lo que ofrece información sobre la igualdad de oportunidades (GRI, 2013a). Este indicador se concreta en la siguiente información:

- Distribución del PAS y del PDI por sexo y grupos de edad según categoría profesional.

- Composición de los órganos de gobierno (colegiados y unipersonales), indicando:

- $\quad N^{0}$ de hombres y mujeres.

- \% de mujeres.

- Desglose por grupos de edad.

- Coeficiente de género ${ }^{10}$.

Igualdad de retribución entre mujeres y hombres

No se hacen propuestas para el indicador G4-LA13 al tratarse de una cuestión no aplicable. En el sector de la universidad pública, las retribuciones se hacen atendiendo a criterios legales según colectivo y categoría profesional.

10.- Para su cálculo se tiene en cuenta el porcentaje de mujeres que participan en los órganos de gobierno y representación, citados en el artículo 13 de la Ley Orgánica de Universidades y que son nombrados por designación (no electos), respecto al total de personal designado en dichos órganos (Universidad Internacional de Andalucía, 2012). 


\section{Evaluación de las prácticas laborales de los proveedores}

La evaluación de la cadena de suministro es uno de los nuevos aspectos de la última versión de la Guía GRI (GRI, 2013a). Con estos indicadores se informa los proveedores que se han seleccionado a partir de la debida diligencia sobre prácticas laborales y la medida en que se es consciente de los impactos negativos de las prácticas laborales en la cadena de suministro (GRI, 2013a).

\section{Tabla 14. Indicadores sociales sobre evaluación de las prácticas laborales de los proveedores}

\begin{tabular}{|l|l|}
\hline CÓDIGO G4 & PROPUESTAS DE INDICADORES UNIVERSITARIOS \\
\hline G4-LA14 & $\begin{array}{l}N^{\circ} \text { de contratos de productos, obras o servicios en cuyas prescripciones técnicas se incluyeron criterios } \\
\text { laborales. }\end{array}$ \\
\hline & $-N^{\circ}$ de empresas que han sido evaluadas en sus prácticas laborales o $n^{\circ}$ de informes de empresas sobre \\
& el desempeño de sus prácticas laborales. \\
- Relación de impactos en las prácticas laborales por tipo de empresa. & $-N^{\circ}$ de proveedores con los que se han puesto en marcha acciones para minimizar impactos después \\
& de la evaluación. \\
& $-N^{\circ}$ de proveedores con los que se ha finalizado relación contractual después de la evaluación. \\
& $($ GRI, 2013a) \\
\hline
\end{tabular}

Mecanismos de reclamación sobre las prácticas laborales

El indicador G4-LA16 ${ }^{\mathrm{V}}$, hace referencia a la eficacia de los mecanismos de reclamación sobre prácticas laborales, incluidas las relacionadas con la cadena de suministro. El contenido de este indicador se concreta en los siguientes puntos:

- $\mathrm{N}^{0}$ de reclamaciones recibidas sobre prácticas laborales y \% de reclamaciones resueltas.

- Mecanismos formales de reclamación.

\section{c.2.) Derechos humanos}

La gestión de los derechos humanos trata, en ocasiones, de cuestiones no aplicables o ya superadas (Reyes et al., 2014). En el contexto de las universidades públicas españolas en general y, en concreto, de la UCO, la gestión de derechos humanos contempla el apoyo a iniciativas y productos de Comercio Justo ${ }^{11}$ en su cadena de suministro.

11.- Sistema comercial basado en el diálogo, la transparencia y el respeto, que busca una mayor equidad en el comercio internacional prestando especial atención a criterios sociales y medioambientales. Contribuye al desarrollo sostenible ofreciendo mejores condiciones comerciales y asegurando los derechos de productores/as y trabajadores/as desfavorecidos, especialmente en el Sur (Organización Mundial del Comercio Justo, WFTO). 
Inversión

Para poner de manifiesto cómo se integran los derechos humanos en las decisiones económicas de la universidad, el indicador G4-HR1ii recopila información sobre el número de contratos de suministro de productos en los que se hayan incluido cláusulas o criterios de valoración relacionados con el Comercio Justo. Por su parte, el indicador G4-HR2ii, con el objetivo de visualizar la capacidad de la universidad para poner en práctica sus políticas en materia de derechos humanos, refleja las horas de formación del personal sobre RSU, Responsabilidad Social Empresarial (RSE) y sobre los derechos frente a la administración pública, igualdad y medio ambiente ${ }^{12}$.

\section{No discriminación}

Las universidades españolas no están exentas de elementos que alteran la igualdad de oportunidades (Escolano, 2006). Por ello es importante informar acerca de los mecanismos a disposición de la comunidad universitaria para informar sobre incidentes de discriminación y el tratamiento que reciben dichas comunicaciones. El indicador G4-HR3ii es el encargado de reflejar esta información a través del número de incidentes de discriminación registrados por el Defensor Universitario, Servicio de Personal y Unidad de Igualdad, indicando fecha de registro de las incidencias y estado de las mismas (caso analizado, plan correctivo en marcha, plan correctivo aplicado y revisión de resultados, expediente cerrado) (GRI, 2013a).

\section{Libertad de asociación y negociación colectiva, trabajo infantil y trabajo forzoso}

Los aspectos tratados en los indicadores G4-HR4 ${ }^{i i}$, G4-HR5 ${ }^{\mathrm{ii}}$ y G4-HR6ii, se centran en la evaluación de proveedores de determinados productos susceptibles de ser fabricados en áreas geográficas en las que los derechos humanos no siempre están garantizados. La información relativa a estos tres indicadores se centra en el número de análisis de productos (ejemplo: suministros como alimentación, textil, equipos informáticos, etc.) en relación a su proceso de fabricación realizados a través de consultas a proveedores.

\section{Medidas de seguridad}

El comportamiento del personal de seguridad hacia terceros respecto al uso de la fuerza se basa en su formación sobre derechos humanos. El indicador G4-HR7ii recoge información sobre:

- $\%$ del personal de seguridad que ha sido formado en materia de derechos humanos.

- Inclusión de cláusulas en el contrato del servicio de seguridad que hagan referencia a la formación del personal en esta materia.

12.- Se puede considerar la temática de la formación relacionada con el resto de derechos humanos identificados en la Tabla de Derechos Humanos 2.0 Cuadro de normas y mecanismos para la Protección de los Derechos Humanos. Disponible en http://www.derechoshumanos.net/derechos/ 


\section{Derechos de la población indígena}

No se hacen propuestas para el indicador G4-HR8 al no resultar de aplicación para la UCO puesto que en su ámbito geográfico no hay comunidades indígenas.

\section{Evaluación}

El indicador G4-HR9 recoge información sobre el número de centros que han sido objeto de examen en materia de derechos humanos o en los que se ha evaluado el impacto en los derechos humanos, desglosado por país. Por el momento, no se dispone de ejemplos de indicadores similares en otras universidades ni de información suficiente para hacer propuestas que respondan a los requisitos del GRI o para afirmar que este indicador no es aplicable a la actividad de la UCO.

\section{Evaluación de los proveedores en materia de derechos humanos}

El indicador G4-HR10 ii informa sobre los proveedores que se han seleccionado o contratado a partir de procesos de diligencia debida sobre derechos humanos, considerando el número de informes de evaluación de proveedores en los que se haga referencia al suministro de productos de Comercio Justo.

Por su parte, el indicador G4-HR11 ${ }^{\mathrm{V}}$, informa sobre la medida en que se es consciente de los impactos negativos de la cadena de suministro desde la perspectiva de los derechos humanos (GRI, 2013a) a través de los siguientes ítems:

- $\mathrm{N}^{0}$ de empresas que han sido evaluadas en sus medidas de seguridad.

- $\quad \mathrm{N}^{0}$ de proveedores con los que se han puesto en marcha acciones para minimizar impactos después de la evaluación.

- $\quad \mathrm{N}^{\circ}$ de proveedores con los que se finalizado relación contractual después de la evaluación.

Mecanismos de reclamación en materia de derechos humanos

La información sobre la eficacia de los mecanismos universitarios resolver conflictos en materia de derechos humanos dentro de la propia universidad y en su relación con proveedores, viene concretado en el indicador G4-HR12ii a través de los siguientes ítems:

- Mecanismos formales de reclamación sobre derechos humanos.

- Manifestación sobre la existencia o no de reclamaciones sobre derechos humanos y \% de reclamaciones resueltas.

\section{c.3.) Sociedad}

Según la Guía G4 (GRI, 2013a), este apartado aborda la repercusión que la organización, en nuestro caso la UCO, tiene en la sociedad y en las comunidades locales. 


\section{Comunidades locales}

Una correcta gestión de los impactos sobre la sociedad en la que se desarrolla la actividad universitaria implica un análisis de las expectativas y necesidades locales, así como una planificación y evaluación de los programas implantados (GRI, 2013a). Dicha evaluación se refleja en los indicadores G4-SO1 iii y el G4-SO2. El primero de ellos recopila información sobre:

- $\quad N^{0}$ de ayudas para la realización de actividades culturales e importe (en €).

- $\quad N^{0}$ de proyectos culturales presentados y $n^{0}$ de proyectos dotados de ayudas.

- Colaboraciones con instituciones culturales.

- Actividades culturales llevadas a cabo.

- $\mathrm{N}^{0}$ de usuarios de instalaciones.

- $\mathrm{N}^{0}$ de tarjetas deportivas y \% de tarjetas pertenecientes a la comunidad universitaria.

- Eventos deportivos.

- Donaciones diferentes a las dirigidas a la comunidad universitaria (cantidades, finalidad, objetivos).

- Colaboraciones con distintos colectivos (actividad del colectivo y naturaleza de la ayuda)

- Recursos, organización, actividades e impactos relacionados con el Voluntariado y la Participación social.

- Recursos, organización, actividades e impactos relacionados con la migración y la interculturalidad, la igualdad y la cooperación internacional.

- Proyectos de cooperación al desarrollo y presupuesto total (indicando origen de la financiación)

- $\quad \mathrm{N}^{0}$ de participantes PDI en acciones de cooperación.

- Actividades de difusión del conocimiento.

- Cátedras universidad-empresa

- Fundaciones universidad-empresa

- Transferencia del conocimiento ( $n^{0}$ convenios con empresas e instituciones)

- Ayudas para transferencia del conocimiento (importe y origen)

Por su parte, para el indicador G4-SO2 no hay ejemplos similares en otras universidades ni se dispone de información suficiente para hacer propuestas que respondan a los requisitos del GRI o para afirmar que no es de aplicación a la actividad de la UCO. Sin embargo, se puede realizar un análisis de los impactos tanto positivos como negativos que la actividad de los centros universitarios tienen en las comunidades locales en las que se encuentran (asociaciones de vecinos, agrupaciones de empresarios de la zona, etc.).

\section{Lucha contra la corrupción}

La corrupción en la administración pública, al igual que en el sector privado, ocurre en entornos en los que la rendición de cuentas es limitada y existe escasa transparencia en las operaciones de gobierno (Diego, 2006). Por ello es importante para una universidad pública como la UCO comunicar información acerca de los siguientes indicadores de la Tabla 15. 


\section{Tabla 15. Indicadores sociales sobre la lucha contra la corrupción}

\begin{tabular}{|l|l|}
\hline CÓDIGO G4 & PROPUESTAS DE INDICADORES UNIVERSITARIOS \\
\hline G4-SO3 & $\begin{array}{l}\text { - Mecanismos de auditoría y transparencia en la gestión económica, indicando a qué servicios u órganos } \\
\text { afectan (gestión económica, contratación, gestión de personal, sindicatos, etc.) }\end{array}$ \\
& $\begin{array}{l}\text { - Identificación de organismos responsables y herramientas para el seguimiento y medición del cumplimiento } \\
\text { normativo y el control de la financiación (memorias económicas y auditorías, protocolo de seguimiento del } \\
\text { Plan Estratégico, sistemas de evaluación, etc.) } \\
\text { - Manifestar si existen o no evidencias de prácticas corruptas. }\end{array}$ \\
\hline G4-SO4ii & $\begin{array}{l}\text { Horas de formación al personal sobre: } \\
\text { - RSU y RSE } \\
\text { - Ley 19/2013, de 9 de diciembre, de transparencia, acceso a la información pública y buen gobierno. }\end{array}$ \\
\hline G4-SO5 ii & Evaluación del cumplimiento con la legislación en materia de gestión económica, financiera y presupuestaria. \\
\hline
\end{tabular}

ii) La UCO no comunica, por el momento, información relativa a este indicador.

\section{Política pública}

El indicador G4-SO6 6 ii pretende aportar transparencia en las relaciones y actividades universitarias vinculadas a causas políticas. Por ello, se debe informar acerca de la realización o no de pagos a partidos políticos y organizaciones similares, así como del valor de las contribuciones (en su caso).

Prácticas de competencia desleal

La legislación que regula las prácticas monopolísticas y la libre competencia son de aplicación a aquellas que puedan afectar al mercado nacional. La actividad universitaria pública queda fuera del mercado por lo que el indicador G4-SO7 no es de aplicación en este caso.

\section{Cumplimiento regulatorio}

Además de informar acerca del cumplimiento regulatorio en materia de medio ambiente (indicador G4-EN29), la UCO debe informar acerca del importe (en €) de las multas por incumplimiento de normativas o legislación de aplicación en otros ámbitos. Si no existen, debe informar con una breve declaración. Esta es la información que se recoge en el indicador G4-SO8vi .

\section{Evaluación de la repercusión social de los proveedores}

Tanto el indicador G4-SO9, que informa sobre el porcentaje de proveedores que se han seleccionado a partir de la debida diligencia sobre aspectos sociales, como el G4-SO10 que informa sobre la medida en que se es consciente de los impactos sociales negativos en la cadena de suministro, aparece por primera vez en la versión G4 de la Guía del GRI (GRI, 2013a). Para la UCO, estos indicadores se concretan en los puntos mostrados en la Tabla 16. 


\section{Tabla 16. Indicadores sociales sobre la evaluación de la repercusión social de los proveedores}

\begin{tabular}{|l|l|}
\hline CÓDIGO G4 & PROPUESTAS DE INDICADORES UNIVERSITARIOS \\
\hline G4-SO9 & $\begin{array}{l}N^{\circ} \text { de contratos de productos, obras o servicios en cuyas prescripciones técnicas se incluyeron criterios } \\
\text { sociales. }\end{array}$ \\
\hline & $\begin{array}{l}-N^{0} \text { de empresas que han sido evaluadas desde el punto de vista social o } n^{\circ} \text { de informes de evaluación } \\
\text { de empresas. } \\
- \text { Relación de aspectos sociales considerados en la evaluación. } \\
-N^{\circ} \text { de proveedores con los que se han puesto en marcha acciones para minimizar impactos después de la } \\
\text { evaluación. } \\
-N^{0} \text { de proveedores con los que se ha finalizado relación contractual después de la evaluación. } \\
(\text { GRI, 2013a) }\end{array}$ \\
\hline
\end{tabular}

Mecanismos de reclamación por impacto social

Para evaluar la eficacia de los mecanismos de reclamación puestos en marcha por la universidad, el indicador G4-SO11v se concreta en los siguientes ítems:

- $\mathrm{N}^{0}$ de reclamaciones recibidas sobre impactos sociales y \% de reclamaciones resueltas.

- Mecanismos formales de reclamación.

\section{c.4.) Responsabilidad sobre productos}

Este apartado de la Guía aborda aspectos relacionados con los productos y servicios que afectan directamente a los grupos de interés, más en concreto, a los clientes (GRI, 2013a). Para la UCO, cuya actividad se centra en la docencia, investigación y la gestión, no son de aplicación los indicadores estrictamente relacionados con actividades productivas. En cambio, sí le son de aplicación aquellos indicadores asociados a servicios. En nuestro caso, entendemos como cliente a todos los usuarios de las instalaciones universitarias.

\section{Salud y seguridad de los clientes}

El objetivo que persigue el GRI con este apartado es identificar la existencia, el alcance y la eficacia de los mecanismos de control relacionados con la salud y la seguridad en el desarrollo de los servicios que ofrece la organización (GRI, 2013a). Para la UCO, dicho objetivo se evalúa a través de los siguientes indicadores:

- G4-PR1 iii: $N^{0}$ de evaluaciones de instalaciones en materia de seguridad y salud.

- G4-PR2 ${ }^{\mathrm{ii}}$ : No de incidencias registradas en relación a seguridad y salud de los alumnos o usuarios de instalaciones universitarias. Si no existen, informar con una breve declaración. 


\section{Etiquetado de los productos y servicios}

Para la UCO, los indicadores de este apartado (ver Tabla 17) se centran en la importancia de la disponibilidad de información acerca de las características de los servicios prestados (entendiendo la docencia también como un servicio) y la posibilidad del usuario de comunicar discrepancias entre la información facilitada y el servicio real que recibe.

\section{Tabla 17. Indicadores sociales sobre información de productos y servicios}

\begin{tabular}{|c|c|}
\hline CÓDIGO G4 & PROPUESTAS DE INDICADORES UNIVERSITARIOS \\
\hline G4-PR3ii & $\begin{array}{l}\text { - Referencia a la participación de la universidad en el Sistema Integrado de Información Universitaria. } \\
\text { - Referencia a la disponibilidad de los procedimientos de evaluación de asignaturas en la página web } \\
\text { de la universidad. } \\
\text { - Referencia a la guía para la elaboración de cartas de servicios universitarios (enlace al documento) } \\
\text { como procedimiento relativo a la información de los mismos. }\end{array}$ \\
\hline G4-PR4 ${ }^{\mathrm{ii}}$ & $\begin{array}{l}\text { № de expedientes relacionados con el incumplimiento de la regulación legal o de códigos voluntarios relativos } \\
\text { a la información de servicios a través de la memoria del Defensor Universitario y Gerencia (p. ej., } \\
\text { procedimientos de evaluación de asignaturas o exámenes, procedimientos de gestión universitaria, etc.). } \\
\text { Si no existen, informar con una breve declaración. }\end{array}$ \\
\hline G4-PR5 5iii & $\begin{array}{l}\text { - Indicadores asociados a la evaluación de la calidad de la docencia (sistemas de planificación, seguimiento } \\
\text { y evaluación de la oferta académica, encuestas de satisfacción del alumnado) } \\
\text { - Mecanismos de evaluación e índice de satisfacción del profesorado con la docencia impartida. } \\
\text { - Evaluación general de los estudiantes, del PDI, del PAS y de usuarios externos en relación a distintos } \\
\text { aspectos del funcionamiento y servicios de la universidad. }\end{array}$ \\
\hline
\end{tabular}

ii) La UCO no comunica, por el momento, información relativa a este indicador.

iii) La UCO informa sobre este indicador aunque no lo identifica como tal.

\section{Comunicaciones de mercadotecnia}

El indicador G4-PR6 informa sobre si la organización vende productos prohibidos o en litigio. La actividad de la UCO no pertenece al sector productivo ni tiene como objetivo principal la venta de productos, por lo que no resulta de aplicación y no se hacen propuestas.

Por otra parte, el indicador G4-PR7ii está relacionado con las comunicaciones publicitarias de los servicios que la UCO desempeña y recoge información sobre el número de incidencias sobre discrepancias entre la programación prevista para asignaturas, circunstancias de su impartición o evaluación, tutorías, información y publicidad de becas y uso de infraestructuras. Si no existen, se debe informar con una breve declaración. 


\section{Privacidad de los clientes}

Entendiendo como cliente todo aquel usuario de las instalaciones universitarias, el indicador G4PR8iï hace referencia a:

- Las medidas para garantizar la seguridad de los datos personales que custodia la universidad.

- $\quad \mathrm{N}^{0}$ de incumplimientos identificados en la memoria del Defensor Universitario o en Gerencia.

- $\mathrm{N}^{0}$ de reclamaciones referidas a la universidad presentadas ante la Agencia Española de Protección de Datos. Si no existen, informar con una breve declaración.

\section{Cumplimiento regulatorio}

Al igual que para los aspectos ambientales, la UCO debe informar a través del indicador G4-PRgii acerca del número de multas o sanciones por incumplimiento de normativas sobre los servicios prestados. Si no existen, se debe informar con una breve declaración.

\section{4.- Discusión}

El presente trabajo ha desarrollado una propuesta de indicadores de RS adaptados a la Guía G4 del GRI (GRI, 2013a) y a la propia realidad de la UCO, como objeto del estudio. De hecho, este trabajo es una herramienta práctica que puede ayudar al resto de universidades públicas a superar la barrera de falta de indicadores identificada por Larrán et al. (2012) para el desarrollo de estrategias de RSU, así como a ajustar el contenido de sus memorias de RS a esta nueva realidad del GRI (la versión G4 de la Guía) en las líneas económica, ambiental y social.

Del análisis de los datos con respecto al estudio de caso, se confirma la mayor atención, en la memoria de RSU de la UCO, a indicadores ambientales y económicos (Lozano, 2011) y una menor proporción de indicadores sociales con respecto al total de indicadores propuestos por el GRI en cada ámbito.

Tal y como exponen Reyes, Montes y Laguna (2014), los indicadores de desempeño propuestos por la Guía asociados a la gestión de derechos humanos o a la responsabilidad del producto son de difícil aplicación al contexto universitario. En cambio, a la hora de hacer nuestra propuesta, hemos comprobado que sí se puede aportar información acerca de los indicadores ambientales no relacionados directamente con la actividad universitaria (gestión de residuos, biodiversidad, etc.) 
Siguiendo con el principio de materialidad de la Guía del GRI (GRI, 2013b), uno de los pasos fundamentales a la hora de seleccionar el contenido de una memoria de sostenibilidad es la identificación de los aspectos que son de relevancia para la organización o sus grupos de interés. En relación a esta tarea, los propios grupos de interés tienen un papel muy importante en la determinación de la relevancia de la información transmitida (Toppinen y Korhonen-Kurki, 2013). La UCO, gracias a su participación en el Proyecto de Responsabilidad Social en el Sistema Universitario Andaluz (FCCSSA, 2009), ha realizado un análisis de las expectativas de los diferentes grupos de interés universitarios, lo que facilita la identificación de sus aspectos materiales y, por tanto, de los indicadores que debe comunicar para elaborar memorias "de conformidad" al GRI.

Hay que tener en cuenta que los indicadores aquí propuestos vendrían a sumarse a la información que las universidades ya comunican en sus memorias y que constituyen aspectos materiales que el GRI no contempla (compromiso con el alumnado o perspectiva universitaria del compromiso social).

Como limitación a este trabajo, se señala que las propuestas realizadas se basan en una muestra limitada de universidades e informes, por lo que se considera de gran interés continuar con la realización de investigaciones relacionadas con la identificación de aspectos materiales universitarios y la validación de las propuestas a través del diálogo con los grupos de interés y la ampliación de la consulta a expertos.

\section{Anexo 1}

- Las últimas memorias de responsabilidad social de universidades españolas que registran sus informes en la "Sustainability Disclosure Database" en 2011 (GRI, 2011b):

- Memoria 2011/2012 de la Universidad de Cádiz (Universidad de Cádiz, 2012).

- Memoria de Responsabilidad Social 2010/2011 de la Universidad Internacional de Andalucía (Universidad Internacional de Andalucía, 2012).

- Memoria de Responsabilidad Social 2010/2011 de la Universidad de Santiago de Compostela (Universidad de Santiago de Compostela, 2012).

- Memoria de Responsabilidad Social 2011/2012 de la Universidad de Zaragoza (Universidad de Zaragoza, 2012).

- Memoria de Responsabilidad Social 2009/2010 de la Escuela Técnica Superior de Ingenieros Industriales de la Universidad Politécnica de Madrid (Escuela Técnica Superior de Ingenieros Industriales de la Universidad Politécnica de Madrid, 2011). 
- Propuestas a nivel nacional que, tal y como mencionan Moneva y Martín (2012) tratan de integrar las dimensiones educacionales a las memorias de RSU, como son el Proyecto Universidades Responsables (Universidad de Zaragoza, 2007) y el Proyecto de Responsabilidad Social del Sistema Universitario Andaluz (FCCSSA, 2009).

- El informe de 2012 del Ministerio de Educación sobre el sistema universitario español (España. Ministerio de Educación, Cultura y Deporte, 2012)

\section{Bibliografía}

ALDEANUEVA, I. (2011): Responsabilidad Social en la Universidad: estudio de casos y propuesta de despliegue. Tesis doctoral, Facultad de Ciencias Económicas y Empresariales, Universidad de Málaga, España. Recuperado de http://riuma.uma.es/xmlui/handle/10630/4687.

BARAÑANO, M. \& REY, V. (2012): "Concepto, antecedentes y relevancia de la responsabilidad social y del compromiso de las universidades con el desarrollo sostenible". En: M. Barañano (Coord.), La responsabilidad social como misión en las universidades españolas y su contribución al desarrollo sostenible. Diagnóstico y buenas prácticas, Proyecto de investigación financiado por el Ministerio de Educación, Cultura y Deporte, 18-47. Recuperado de http://138.4.83.162/mec/ayudas/repositorio/20121130213510INFORME_RSU_2.pdf.

BENAVIDES, C.A. (Coord.) (2012): Integración de la responsabilidad social en los sistemas de garantía interna de calidad: hacia una universidad saludable, sostenible y solidaria. (EA 2011-0027). Programa de Estudio y Análisis del Ministerio de Educación, Dirección del Plan Estratégico y Responsabilidad Social de la Universidad de Málaga y Cátedra Santander de Responsabilidad Social Corporativa de la Universidad de Málaga, Málaga. Recuperado de http://www.uca.es/recursos/doc/Unidades/Gab_Com_Mark/logosimbolos/Version_2/1205196331_2252013144826.pdf

CASANI, F. \& PÉREZ-ESPARRELL, C. (2009): "La responsabilidad social en las universidades públicas españolas: vectores de cambio en la gobernanza", Investigaciones de Economía de la Educación, 4, 127-137.

CONFERENCIA DE RECTORES DE LAS UNIVERSIDADES ESPAÑOLAS (2012): Directrices para la introducción de la Sostenibilidad en el Curriculum. Recuperado de: http://www.crue.org/Sostenibilidad/CADEP/Documents/DIRECTRICESSOSTENIBILIDAD\%20CRUE\%2 02012.pdf 
DE LA CUESTA, M. \& VALOR, C. (2003): "Responsabilidad social de la empresa. Concepto, medición y desarrollo en España", Boletín Económico De ICE, Información Comercial Española, 2755, 7-20.

DE GOBROIS, D. (2012): "Corporate social responsibility reporting by the global hotel industry: commitment, initiatives and performance", International Journal of Hospitality Management, 31(3), 896905.

DE LA RED, N. (2009): "Necesidades emergentes y responsabilidad social universitaria", Alternativas. Cuadernos de Trabajo Social, 16, 65-76.

DE TORO, A., GOMERA, A., AGUILAR, J.E., GUIJARRO, C., ANTÚNEZ, M., VAQUERO, M. (2015): La huella de Carbono de la Universidad de Córdoba 2014, Universidad de Córdoba, Córdoba. Recuperado de http://www.uco.es/servicios/dgppa/images/sepa/huellaC2014.pdf

DIEGO, O. (2006): La ética y la corrupción en la política y la administración pública. (Tesis de maestría), Universidad Internacional de Andalucía, España.

ESCARDÍBUL, J. \& PÉREZ, C. (2013): "La financiación de las universidades públicas españolas. Estado actual y propuestas de mejora", Revista de Educación y Derecho. Education and Law Review, 8.

ESCOLANO, E. (2006): "Discriminación en un medio meritocrático: las profesoras en la universidad española", Revista Mexicana de Sociología, 68(2), 231-263. Recuperado de http://www.jstor.org/stable/20454234

ESCUELA TÉCNICA SUPERIOR DE INGENIEROS INDUSTRIALES DE LA UNIVERSIDAD POLITÉCNICA DE MADRID (2011): Memoria de Responsabilidad Social. Curso 2009/2010, Autor, Madrid. Recuperado de http://es.scribd.com/doc/76209054/Memoria-RS-Escuela-Industriales-UPM

ESPAÑA. MINISTERIO DE EDUCACIÓN, CULTURA Y DEPORTE (2012): Datos y cifras del sistema universitario español. Curso 2012-2013. Recuperado de http://www.mecd.gob.es/dctm/sue/datosy-cifras-sistema-universitario-espanol.pdf

FORÉTICA (2016): Informe Responsabilidad Social en las universidades: Del conocimiento a la acción. Pautas para su implantación. Recuperado de http://www.foretica.org/informe_ejecutivo_rsu.pdf

FORO DE LOS CONSEJOS SOCIALES DE LAS UNIVERSIDADES PÚBLICAS DE ANDALUCÍA (2009): Responsabilidad Social del Sistema Universitario Andaluz, Recuperado de http://rsuniversitaria.org/web/images/stories/librocompleto.pdf

GANGA, F. \& NAVARRETE, E. (2012): "Universidades privadas y su responsabilidad social en Chile: Un estudio exploratorio", Opción, 28(68), 243-256. Recuperado de http://www.redalyc.org/articulo.oa?id=31025437012

GLOBAL REPORTING INITIATIVE (2011a): Guía para la elaboración de memorias de sostenibilidad. Versión 3.1. Recuperado de https://www.globalreporting.org/resourcelibrary/Spanish-G3.1Complete.pdf 
GLOBAL REPORTING INITIATIVE (2011b): Sustainability Disclosure Database. Recuperado de http://database.globalreporting.org/

GLOBAL REPORTING INITIATIVE (2013a): Guía para la elaboración de memorias de sostenibilidad. Versión 4. Manual de aplicación. Recuperado de https://www.globalreporting.org/resourcelibrary/Spanish-G4-Part-Two.pdf

GLOBAL REPORTING INITIATIVE (2013b): Guía para la elaboración de memorias de sostenibilidad. Versión 4. Principios de elaboración de memorias y contenidos básicos, Recuperado de https://www.globalreporting.org/resourcelibrary/Spanish-G4-Part-One.pdf

GLOBAL REPORTING INITIATIVE (2013c): Overview of changes in standard disclosures from G3.1 to G4 guidelines. Recuperado de https://www.globalreporting.org/resourcelibrary/GRI-G4-OverviewTables-G3.1-vs-G4.pdf

HART, S.M. (2013): "The crash of Cougar flight 491: a case study of offshore safety and corporate social responsibility", Journal of Business Ethics, 113(3), 519-541.

LARRÁN, M., DE LA CUESTA, M., FERNÁNDEZ, A., MUÑOZ, M.J., MONEVA, J.M., RODRÍGUEZ, J., MARTÍN, E., CALZADO, Y., HERRERA, J., ANDRADES, J. \& GRADE, R. (2012): Análisis del nivel de implantación de políticas de responsabilidad social en las universidades españolas, Fundación Carolina, Madrid. Recuperado de https://www.carm.es/web/servlet/integra.servlets.Blob?ARCHIVO=RESULTADOS\%20PRO YECTO\%20CAROLINA. pdf\&TABLA=ARCHIVOS\&CAMPOCLAVE=IDARCHIVO\&VALORCLAVE=80437\&CAMPOIMAGEN=ARCHIVO\&IDTIPO=60\&RASTRO=c2126\$m34533

LOZANO, R. (2006): "A tool for a Graphical Assessment of Sustainability in Universities (Gasu)", Journal of Cleaner Production, 14(9-11), 963-972.

LOZANO, R. (2011): "The state of sustainability reporting in universities", Journal of Sustainability in Higher Education, 12, 67-78.

MONEVA, J.M. \& LLENA, F. (1996): "Análisis de la información sobre responsabilidad social en las empresas industriales que cotizan en bolsa", Revista Española de Financiación y Contabilidad, 25(87), 361-401. Recuperado de http://dialnet.unirioja.es/descarga/articulo/44209.pdf

MONEVA, J.M. \& MARTÍN, E. (2012): "Universidad y desarrollo sostenible: Análisis de la rendición de cuentas de las universidades públicas desde un enfoque de responsabilidad social", Revista Iberoamericana de Contabilidad de Gestión, 10(19). Recuperado de http://www.observatorio-iberoamericano.org/RICG/N\%C2\%BA_19/Jos\%C3\%A9_Mariano_Moneva_y_Emilio_Mart\%C3\%ADn_Vall esp\%C3\%ADn.pdf

MUSTATA, R.V., BONACI, C.G., HINTEA, C. \& NEAMTU, B. (2013): "Business education for sustainable development: the case of romanian universities", Amfiteatru Economic, 15(7), 802-818.

NACIONES UNIDAS (2008): Orientación sobre los indicadores de la responsabilidad de las empresas en los informes anuales. Recuperado de http://unctad.org/es/docs/iteteb20076_sp.pdf 
ORELLANA, A.R. (2006): "La RSC y la triple cuenta de resultados", Estrategia financiera, 231, 56-63.

PEREA-SARACHAGA, J. (1995): "La diligencia debida (due diligence) medioambiental en transacciones empresariales", DYNA, 70(8), 18-20.

REYES, L., MONTES, P. \& LAGUNA, P. (2014): "De La Responsabilidad Social Pública a la Responsabilidad Social Universitaria: Un estudio de la certificación de memorias para el caso español". En: I Jornadas Internacionales sobre Responsabilidad Social Universitaria, Cádiz.

RODRÍGUEZ, J.M. (2010): "Responsabilidad social universitaria: del discurso simbólico a los desafíos reales". En: M. de la Cuesta, C., de la Cruz, C. \& J. M. Rodríguez (coords.), Responsabilidad Social Universitaria, Netbiblo, La Coruña, 3-24.

SAMANIEGO, C. (1998): "Absentismo, rotación y productividad". En: A. Rodríguez (coord.), Introducción a la Psicología del Trabajo y las Organizaciones, Ediciones Pirámide, Madrid, 247256.

TOPPINEN, A. \& KORHONEN-KURKI, K. (2013): "Global Reporting Initiative and social impact in managing corporate responsibility: a case study of three multinationals in the forest industry", Business Ethics: A EuropeanReview, 22(2), 202-217.

UNIVERSIDAD DE CÓRDOBA (2013): Memoria de Responsabilidad Social Universitaria 2011/2012, Autor, Córdoba. Recuperado de http:/www.uco.es/memoria-RSU/2011-2012/memoria.pdf

UNIVERSIDAD DE CÓRDOBA (2014): Memoria de Responsabilidad Social Universitaria 2012/2013, Autor, Córdoba. Recuperado de http://www.uco.es/memoria-RSU/2012-2013/memoria.pdf

UNIVERSIDAD DE CÁDIZ (2012): Memoria 2011/2012, Aurtor, Cádiz. Recuperado de http://memoria.uca.es/descargas/memoria-uca-2011-2012.pdf

UNIVERSIDAD INTERNACIONAL DE ANDALUCÍA (2012): Memoria de Responsabilidad Social 2010/2011. Recuperado de

http://www.unia.es/images/stories/Resp_Social/21x21_memoria_rs_10_11_digital_1a1_02.pdf

UNIVERSIDAD DE SANTIAGO DE COMPOSTELA (2012): Creando valor para la sociedad. Memoria de Responsabilidad Social 2010/2011, Recuperado de

http://www.usc.es/estaticos/calidade/mrs201011/mrs/MRS_ES_2010-2011.pdf

UNIVERSIDAD DE ZARAGOZA (2007): Promoviendo un modelo de Responsabilidad Social en la Universidad de Zaragoza. Universidades Responsables. Recuperado de http://rsuniversitaria.org/web/images/stories/informe\%20UNIVERSIDAD\%20ZARAGOZA.pdf

UNIVERSIDAD DE ZARAGOZA (2012): Memoria de Responsabilidad Social. Curso 2011/2012. Recuperado de http://www.unizar.es/sg/doc/MEMORIA11-12PortadayMemoria.pdf

VALLAEYS, F. (2008): "Responsabilidad social universitaria: una nueva filosofía de gestión ética e inteligente para las universidades", Educación Superior y Sociedad, 2, 191-220. Recuperado de http://ess.iesalc.unesco.org.ve/index.php/ess/article/view/47 
VALLAEYS, F., DE LA CRUZ, C. \& SASIA P. (2009): Responsabilidad Social Universitaria. Manual de primeros pasos, Mc Graw-Hill: México. Recuperado de http://blog.pucp.edu.pe/media/410/20110119-VERSION\%20DIGITAL.pdf

VALAREZO, K. \& TÚÑEZ, J.M. (2014): "Responsabilidad Social Universitaria. Apuntes para un modelo", Revista de Comunicación, 13, 84-117.

Recuperado de http://udep.edu.pe//comunicacion/rcom/pdf/2014/Art084-117.pdf

VEGA, J.L. (2009): Responsabilidad social y los principios del desarrollo sostenible como fundamentos teóricos de la información social de la empresa, ESIC Editorial, Madrid.

VILLARREAL, O. \& LANDETA, J. (2010): "El estudio de casos como metodología de investigación científica en dirección y economía de la empresa. Una aplicación a la internacionalización", Investigaciones Europeas de Dirección y Economía de la Empresa, 16(3), 31-52. Recuperado de http://www.aedem-virtual.com/articulos/iedee/v16/163031.pdf

YIN, R.K. (2009): Case study research: Design and methods (4th Ed.), Sage Publications: Thousand Oaks, CA. 